\title{
Bipedal Humanoid Hardware Design: a Technology Review
}

\author{
Grzegorz Ficht $^{1}$ (D) . Sven Behnke ${ }^{1}$
}

Accepted: 8 March 2021 / Published online: 6 April 2021

(C) The Author(s) 2021

\begin{abstract}
Purpose of Review As new technological advancements are made, humanoid robots that utilise them are being designed and manufactured. For optimal design choices, a broad overview with insight on the advantages and disadvantages of available technologies is necessary. This article intends to provide an analysis on the established approaches and contrast them with emerging ones.

Recent Findings A clear shift in the recent design features of humanoid robots is developing, which is supported by literature. As humanoid robots are meant to leave laboratories and traverse the world, compliance and more efficient locomotion are necessary. The limitations of highly rigid actuation are being tackled by different research groups in unique ways. Some focus on modifying the kinematic structure, while others change the actuation scheme. With new manufacturing capabilities, previously impossible designs are becoming feasible.
\end{abstract}

Summary A comprehensive review on the technologies crucial for bipedal humanoid robots was performed. Different mechanical concepts have been discussed, along with the advancements in actuation, sensing, and manufacturing. The paper is supplemented with a list of the recently developed platforms along with a selection of their specifications.

Keywords Humanoid robotics $\cdot$ Kinematic structure $\cdot$ Actuation $\cdot$ Sensors $\cdot 3 D$-printing $\cdot$ Manufacture

\section{Introduction}

Humanoid robotics has come a long way in its development, with progress accelerating in its every branch by the years. What started as basic, yet fascinating automata has quickly grown into a global interdisciplinary research field in just half of a century. The intertwined dependence of theoretical concepts and available technologies is constantly shaping the evolution of humanoid robotics. Current humanoid robots are not the result of a single

This article belongs to the Topical Collection: Topical Collection on Humanoid and Bipedal Robotics

Grzegorz Ficht

ficht@ais.uni-bonn.de

Sven Behnke

behnke@ais.uni-bonn.de

1 Rheinische Friedrich-Wilhelms-Universität Bonn, Friedrich-Hirzebruch-Allee 8, 53115 Bonn, Germany scientific breakthrough, but rather an accumulation of small incremental achievements in the respective utilised technologies. Humanoids are not the sole beneficiary though, as the relation is mutual. More advanced platforms resulted in increased application complexity, which in turn facilitates the creation of novel technological solutions. Due to these advancements, humanoid robots come in different shapes, sizes, and functionality (see Fig. 1). Much work has been done since the early 2000s, where humanoids were more in the fictional domain, with only few capable robots available [1]. With limited actuation capabilities, only smaller platforms were accessible for many researchers. Size is a limiting factor, as smaller robots are unable to meaningfully interact in an environment meant for humans, which is the ultimate goal of developing these robots. On the other hand, larger robots require disproportionally more actuation power-making the task of building such much more difficult [2]. As in nature [3], mediating size and performance is crucial for achieving an optimal middleground. For all intents and purposes, this review focuses on robots that aim to mimic the bipedal mobility of a human, and the recent advancements in technology that enable it. 

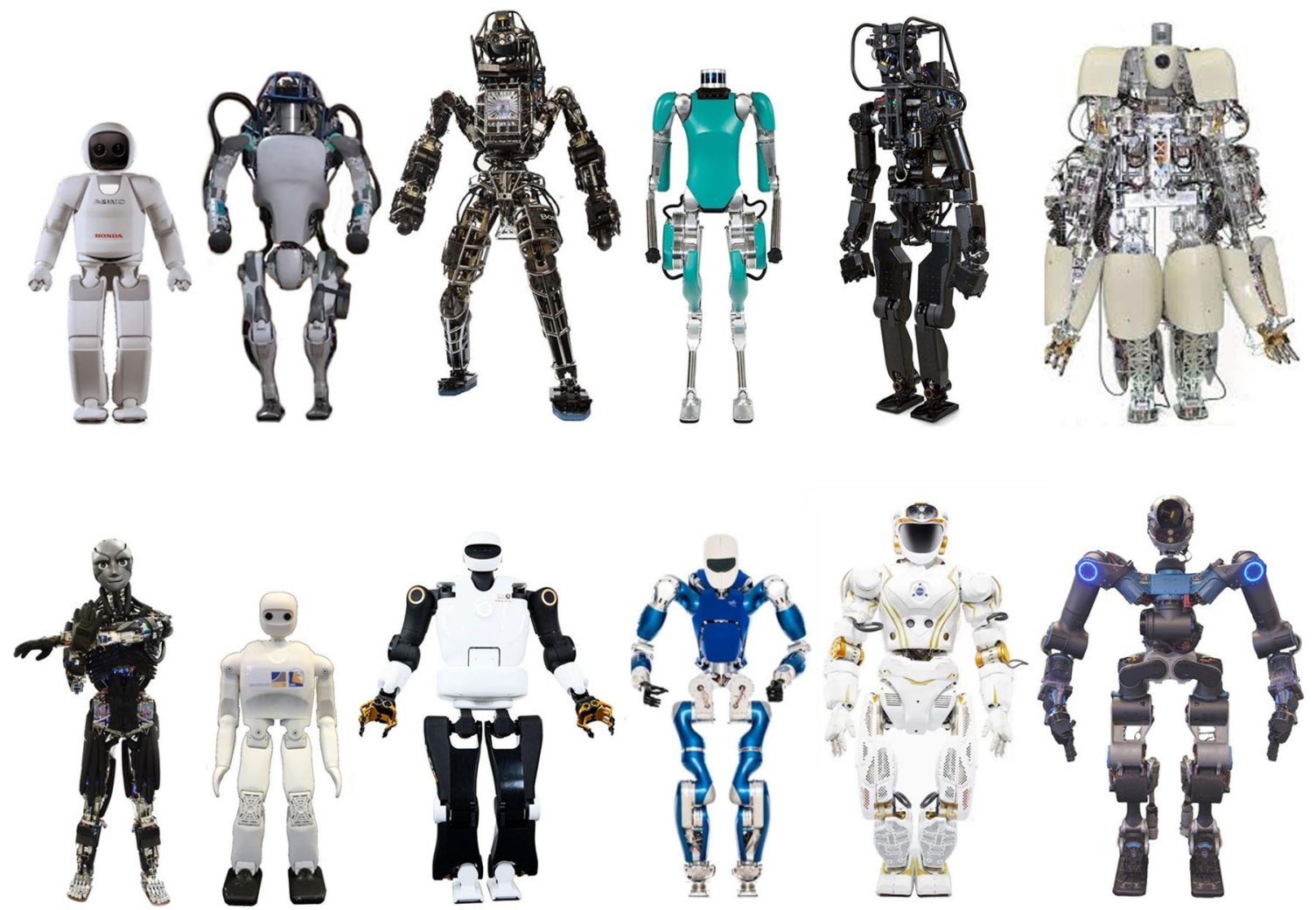

Fig. 1 Most recently developed bipedal humanoid robots with a full humanoid body plan, from left to right, top to bottom: Asimo, Atlas, Atlas-Unplugged, Digit, HRP-5P, Hydra, Kengoro, NimbRo-OP2X, TALOS, Toro, Valkyrie, WALK-MAN

\section{Mechanical Structure}

The defining feature of any bipedal humanoid is its kinematic structure. Initially, the limited technology allowed only for a simplified representation of the humanoid form, often having just the legs. This is not a disadvantage as the design concept might prefer simpler forms, which allow for certain assumptions to be made and exploited in the control part [4]. Perfect examples of this approach are the MIT 3D Biped [5] and ATRIAS [6]. The extending leg with a rotating hip is enough to perform the 3D positioning function without having a human-like form. Both robots uniquely embody the spring-loaded inverted pendulum (SLIP) template for control. The MIT 3D Biped does so with linear thrusters, while ATRIAS has a pair of rotary actuators connected to the anterior and posterior thigh members of a 4-bar parallel linkage through spring plates. Both structures achieve a hip-concentrated mass and low-inertia legs; however, due to the antagonistic actuation regime stemming from the leg design of ATRIAS, a significant amount of energy is wasted [7]. Although impressive, these robots are chiefly used as demonstrators, for exploration of dynamic bipedal locomotion.
For more general applications, robots with a full humanoid body plan have been built. Prominent examples of humanoids such as ASIMO [8], the HRP series [914], HUBO [15], REEM-C [16], and TALOS [17] are all similar in terms of kinematics. The legs are composed of a three-degree-of-freedom (DoF) hip to simulate a spherical joint, one DoF for knee bending and two DoF for an ankle ball joint. As such, six actuators are enough to provide roughly the same form and functionality as a human leg. This structure has the added benefit of enabling a closedform solution for the inverse kinematics. More joints (e.g. a toe) can be introduced to avoid singularities, joint limits, or achieve a specific configuration [18]. The mentioned robots utilise rotary joints with servo-motors in a serial configuration (Fig. 2a). The structure is well-established in the scientific community and has been thoroughly investigated, as evidenced by the number of robots that incorporate it. Its strength lies in the simplified mechanics and control, which comes at the drawback of limited capability. As the rotary actuators are typically placed directly at the joint, the ones closer to the origin of the chain need to carry the ones lower in the chain. This has twofold consequences in backlash or elasticity accumulation 
Fig. 2 Configuration examples of lower leg design: a on-axis serial mechanism, $\mathbf{b}$ off-axis serial mechanism, c crank-lever parallel mechanism, $\mathbf{d}$ linear actuator parallel mechanism, and e mixed serial/parallel mechanism. Grey components represent actuators
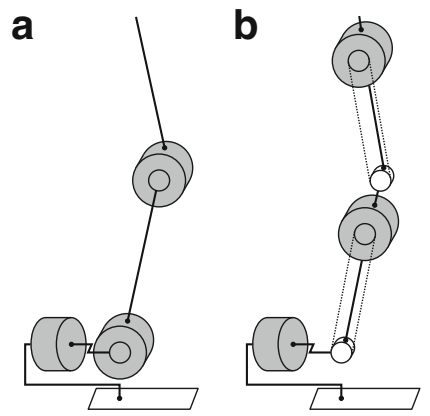

C

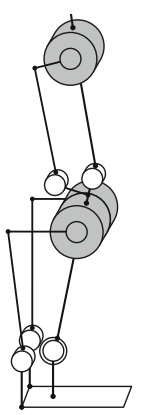

d

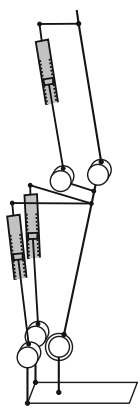

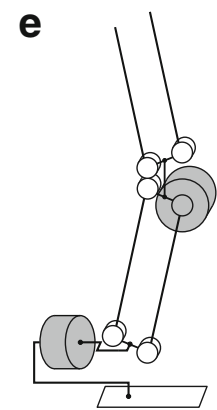

from each joint and increased leg inertia. Both contribute to positioning inaccuracies that the control scheme must compensate for.

Decreased limb rigidity and increased leg inertia can be substantial, which impacts the overall dynamic performance of the system and sets higher requirements for actuators in terms of quality and power output. A remedy to this issue is to attach the actuators off-axis, closest to the root of the link and using a lightweight coupling or transmission (e.g. synchronous belts) as in an earlier version of ASIMO [19] (see Fig. 2b). Placing the actuators off-axis leads to interesting joint designs where parallel linkages can be involved. Parallel mechanisms exhibit higher stiffness due to the mechanical drive coupling, which averages out the actuation error. The downside of parallel solutions is a limited workspace due to the matings of the links as well as an increased mechanical and computational complexity. Some mechanisms can be incorporated for specific joints, and some for whole legs. A Stewart platform type leg [20] might provide benefits in terms of loading capabilities, but the limited range of motion and lack of a knee joint do not translate to human-like capabilities [21].

In terms of human-like joints, A 3-DoF hip is least to benefit from the incorporation of a parallel structure as it is the root of the leg. Decreasing the inertia just marginally requires a significant investment in mechanical development [22]. Due to these mechanical intricacies, the yawing in the hip is still usually performed with a separate rotary actuator [23], with its backlash marginally impacting the positioning of the whole leg. The hip ab/adduction as well as flexion and extension can be performed with two actuators, where the sum of their movements amounts to pitching, while differential movement results in rolling the hip [24]. As a high range of motion in the hip is desirable, only few robots up to date have incorporated parallel linkages in the hip, such as the recent RHP-2 [25]. Implementation of a parallel knee joint is comparably easy, as there is usually sufficient space in the thigh link to place the actuator and transmission. The possible options involve employing either a rotary actuator in a crank- lever mechanism as seen in WALK-MAN [26] and the newer ASIMO [27] or a linear actuator [28], which both pull and push the shank link. In combination with a nontrivial linkage mechanism, the required knee torque can be significantly reduced, leading to a higher torque-to-weight ratio and thus, higher performance [29]. A 2-DoF ankle joint provides the most benefits of using parallel linkages, as the increased stiffness complements a statically stable upright posture without much need for torque expenditure. Similar to the thigh, the shank provides ample space for housing the actuators and couplings, which allow for pitching and rolling the ankle around a Cardan joint. As a result, several compact and elegant solutions have been produced. This includes the crank-lever mechanism found in ASIMO [30], TORO [31], and CogIMon [32] (Fig. 2c), as well as the linear actuator scheme [28, 33, 34] (Fig. 2d).

Apart from joint-specific solutions, few researchers took a different approach to the kinematic structure. Gim et al. [35] designed a hybrid serial-parallel leg, where five of the six leg actuators are located in the hip. This greatly reduces the leg inertia and in turn the required joint velocities and torques, which are also more uniform. The structure has been used to build a Bipedal robot [36], achieving a $15 \%$ decrease in the distance from origin to CoM (scaled to leg length), when compared to a reference robot with a serial kinematic chain [37]. Making assumptions such as walking only on flat ground has allowed for simplifying the control theory [38]. Analogically, this can be taken advantage of in the mechanical part. The NimbRo-OP2(X) robots [39][40•] have adopted a five-DoF serial-parallel leg structure with two 4-bar linkages in the sagittal plane and a serial chain for the lateral direction (Fig. 2e). The 4-bar pantographs constrain the leg orientation, keeping the foot parallel to the waist. When no tilting is present, the feet essentially stay parallel to the ground. The actuation scheme is fundamentally different, where instead of the hip and knee joints, the angle is changed on the thigh and shank. Actuators can be placed at any pantograph axis and synchronised for increased performance. A leg utilising this 
design can also be actuated completely at the hip, reducing the inertia [41], as well as supplemented with a spring to reduce the torque requirements [42].

Biomimetics is a completely separate design concept, which inspires an increasing number of researchers. As humanoid robots are supposed to mimic humans, it becomes natural to utilise not only the concept of a humanoid body plan, but the implementation of it as well. A musculoskeletal structure with biarticular actuation reduces the control bandwidth requirements and allows for coordination and energy transfer between two joints to improve on efficiency [43-45•]. The usage of flexible elements and tendons improves on compliance and shockloading of the limbs [46]. As shown, the co-location of tendons with the skeleton-known as tensegritygreatly reduces the stress on structural parts and allows for using lighter parts, resulting in improved dynamic performance [47]. With multiple tendons going through several joints, minimising the friction or accounting for it becomes necessary [48]. Completely biomimetic humanoids are still scarce. Kenshiro [49], and its successor Kengoro [50] are highly complex humanoids with a humanlike body structure including the skeletal structure and anatomically correct muscle arrangement. However, due to the high complexity in the control and coordination of several 'muscles', the potential of such robots is yet to be explored.

\section{Actuation}

Designing a humanoid robot involves a holistic approach, where the structure of the robot is co-dependent on the chosen actuator and vice-versa. From the mid 1980s, electrical actuators with high-ratio reducers have been the default choice, as they offer a good trade-off between torque, speed, and size. Apart from performance, using electrical actuators has benefits in simplifying the control, due to the nearly linear input-to-output relationship for control and convenient power storage and distribution systems. The high transmission ratio required to provide the necessary torque has a drawback of a high-gain control regime. As humanoid robots are required to interact more and more with their environment, force-feedback control becomes increasingly relevant. This does not cope well with the rigid actuation of the high reduction setup, which interferes with the current/torque relationship and is not robust to shock-loading. This issue is adressed by introducing an elastic element in the structure of the actuator, making it a series elastic actuator (SEA), as used in NASA's Valkyrie [51]. The spring element serves several purposes: it absorbs impacts, stores energy, and controls the output torque through deflection using
Hooke's law. WALK-MAN [52] is equipped with the newest advances in SEA technology, utilising both active and passive adaptation [53]. Existing humanoids can be made compliant, by introducing an elastic element on the output shaft of the actuator [54].

The recent advances in Brushless DC (BLDC) motor manufacturing and control allow for a new approach. Katz [55•], cleverly combines the high torque density of a 'pancake' BLDC motor with a single-stage 1:6 planetary reduction embedded inside the stator bore. As the commutation happens electronically using forward and inverse Park and Clarke transforms, it is possible to sense and control the output torque directly through the Qaxis current. In combination with an embedded impedance controller, these Quasi-Direct Drive (QDD) modules allow for robust, compliant and proprioceptive actuation with high peak power for dynamic locomotion. Although developed for the quadruped MIT Mini Cheetah [56], these actuators can be applied to bipeds as well [57]. Alterations of these actuators (e.g. longer stator, different reduction) with improved specifications are available on the market, making it a matter of time until compliant and dynamically capable humanoid robots become widespread. Electrical motors are typically used for rotary movement; however, they can be used to produce linear motion through a translational (e.g. ball screw) mechanism [28, 34]. They can also be produced as direct drive linear motors, which could be thought as simply 'unrolling' a rotary one on a guideway.

Electrical motors in one form or another are limited in high-power applications through magnetic saturation and thermal limits. The higher the power requirements, the larger the motor. They are also quite fragile, as overloading them or operating in sensitive environments (water, dirt) can quickly lead to them being destroyed. Hydraulics do not suffer from these limitations, as they offer high power density, scale well and can stop under a heavy load without damage even in harsh environments. They are usually produced as pistons, where linear motion is performed by controlling the difference of incompressible liquid (usually oil) between two chambers separated by a movable cylinder. The achievable high-power specification of hydraulics is what attracted roboticists when building the first statically [58] and dynamically moving bipeds [5]. Currently, Boston Dynamics [59] is at the forefront of humanoid robotics using this technology. Their first humanoid PETMAN [60] achieved a humanlike morphology, range of motion, strength and walking. Its direct descendant ATLAS (along with ATLAS-DRC) [61] was built to investigate navigating in unstructured terrain. Highly impressive at the time, both robots showcased the limitation of the hydraulic system: the power storage and distribution were not as easy as with electrical systems, as the robots operated with a tether. Also, every person in the 
vicinity of the robot was required to wear a hearing protector due to the generated noise. This was resolved with ATLASUnplugged, where the hydraulic power unit was upgraded to produce variable pressure set-points, miniaturised, and placed onboard of the robot. This redesign made ATLAS capable of full autonomy and much quieter operation, at the expense of a weight increase from 152 to $182 \mathrm{~kg}$ [61]. The new generation of ATLAS robots [62] is the result of years of exploration and development of mobile hydraulic technology. By miniaturising the whole structure and components (especially the power unit), ATLAS became similar to a human in size $1.5 \mathrm{~m}$ ) and weight $(80 \mathrm{~kg})$, also capable of elegant and powerful human-like movement including gymnastic routines [63] and dancing [64].

The path taken by Boston Dynamics shows that the entry point is steep and requires a synergy of interdisciplinary technical knowledge to take full advantage of the benefits provided by hydraulic actuation. The technology is still unavailable commercially, meaning that any entity interested in using a hydraulic system would face similar problems. During the DARPA Robotics Challenge (DRC) multiple competitors used the ATLAS platform, which was highly robust to not need maintenance for months, but repairs or upgrades had to be done by the manufacturer themselves-halting progress [65]. Pairing the lack of access to technology with the unfamiliarity and inconvenience of working with it (noise, leakages) resulted in very few platforms built up to date. The most recent full-size humanoids include Hydra [66] and TaeMu [67], which differ in the actuation scheme. TaeMu employs the typical central pump scheme with servo valves to regulate the pressure across the robot, where Hydra uses electrohydrostatic actuators (EHAs) [68], each with its own pump. The solution of Hydra combines benefits from both electric and hydraulic actuation: backdrivability, impact resistance, controllability, and power density, but is yet to be optimised for size and weight. In hydraulic systems, oil is still routed through flexible hoses, which reduces joint mobility and leads to leakages over time. Including oil pathways within the structural components of the robots is possible [69], but has to be done at the design level, adding more constraints to the process. As shown, the process of designing and building even the few mentioned humanoids required custom solutions. This might reflect the state of the options available on the market, more precisely the lack of standardised and modular solutions for hydraulically actuated robots.

\section{Sensing}

Similar to humans, humanoids require the sense of selfmovement and environment awareness. Over the years, multiple sensing technologies have been developed and perfected. For body orientation, an inertial measurement unit (IMU) composed of an accelerometer, gyroscope, and magnetometer is typically used. The readings are then fused to provide a full 3D orientation of the body. In combination with a model of the body plan and joint sensors, a full 6D estimation of self-movement can be obtained. Joint position sensing is now usually done with highresolution, hall-effect-based, magnetic encoders. The lack of friction in the sensing element (as in e.g. potentiometers) is a tremendous advantage, which mitigates the need for maintenance. Similarly, torque or force can be estimated by measuring the applied current to the joint, accounting for the transmission losses. In combination with a Jacobian, end-effector wrenches can be estimated. This is sufficient for rough operation, but fine and detailed manipulation relies on accurate force-torque $(\mathrm{F} / \mathrm{T})$ sensor technology. A feedback loop designed to maintain a safe force, constantly applied to the manipulated object is necessary for a vast majority of applications such as opening a bottle or pushing a cart as shown by ASIMO [8]. In locomotion, the stability assessment is also done using an estimation of the center of pressure (CoP) obtained by $\mathrm{F} / \mathrm{T}$ sensors. This puts a requirement on them being robust enough to not only carry the weight of the robot but also endure ground impact forces during walking. The drawback of currently available $\mathrm{F} / \mathrm{T}$ sensors is that the measurements are done using transducers (e.g. strain gauges), with a direct coupling of tension induced by forces going through the sensor. This is also the reason for problems such as long-term deformations (creep) and overloading. Recalibration can prolong the usability of a sensor, unlike overloading caused by unpredictable shock impacts that simply destroy it. It is essential for new generations of F/T sensors to decouple the force from tension, and instead rely on contactless displacement [70] [71], allowing to make the sensors more robust.

For more elaborate multi-contact interactions, endeffector wrench estimation through F/T sensors or Jacobian methods is insufficient. Humans interact with the environment through skin, which allows for sensing various physical quantities such as temperature, humidity, and pressure. It allows for distinguishing different types of contact, its amplitude as well as anticipating it through proximity sensing with hairs. There is a clear demand for full-body robot skin [72] and although skin modules for robotics exist [73], the technology is not mature enough to allow seamless integration with existing robots. The most advanced work in this regard is HEX-o-SKIN [74, 75] incorporating force, temperature, proximity, and acceleration sensors within an inexpensive, scalable, and robust package. The wide variety of applications developed with these modules [76-80•] underlines the importance of full-body skin. Full-body skin 
will also be essential in water-proofing future humanoids for outdoor applications.

\section{Manufacturing}

Manufacturing materials and techniques for robots matter as much as the technology utilised in them. Choosing the proper materials for the structure impacts performance in terms of weight, rigidity, and maintenance. As seen in Table 1 metal alloys are the default choice in most humanoids, which can be attributed to the high rigidity, machinability and heat dissipation capabilities. For this, either subtractive (CNC-milling) or additive manufacturing (casting) is used, each with its benefits and drawbacks. The iCub humanoid [81] is a prime example of a robot constructed with a subtractive process, where a great majority of the 5000 parts is milled [82]. The possible achievable shape is generally limited with milling, as the cutting tool needs to be able to execute a programmed path. 5-axis machines offer the highest fidelity; however, enclosed parts and those with inner structures still need to be made out of several sub-components. Casting can partially alleviate this problem, depending on the part complexity on a case-to-case basis. As shown on LOLA [83], casting allows to achieve quite sophisticated metal parts otherwise unachievable through milling, but requires a mold to be prepared for every part. In research, the low quantity requirements result in low cost-effectiveness of this method.

Another option enhancing the production capabilities was added just recently, when consumer 3D printers became affordable and widespread [84]. 3D-printing allows for fast production times of highly complex parts and an easy transition from prototyping to production. In short time, several 3D-printed humanoid robots of various sizes have been designed and built [39, 40•, 85-87]. The freedom presented through 3D-printing can be exploited either by an expert fine-tuning design details or by algorithms. Topology optimization can produce efficient shapes depending on user criteria (e.g. weight, rigidity) in the presence of constraints such as shape and material properties [83, 88, 89]. There are still some issues when preparing the design files for printing. Due to the additive manufacturing process, the inner structure of the part is not uniform, unlike in subtractive manufacturing. This makes using the currently available stress analysis tools of CAD software ineffective, especially for deposition-based printing. Depending on the type and quality of the printer, the accuracy of the finished print may vary greatly. The number of variables in the process is simply larger, such as the material used, the temperature, fill pattern, or even orientation in which the part is printed. Ideally, the designed components should be disconnected from the production process, in 3D-printing however, they are very much dependent on it. Having in mind these issues and the limitations of the used printer, prototyping of a 3D-printed part is mostly done through iteration. Although there is continuous improvement in software and hardware capabilities of the printers, the deposition process is still limited. For production purposes, using a higher quality process such as selective laser sintering (SLS) can further increase the quality of the parts, making them more rigid and uniform in structure. The process is quite cost-effective for small production batches, after functional prototypes have already been produced. Aside from these polymer-based methods, 3D metal printing is already in advanced development [90], and has the possibility to completely revolutionise production of more than just robots.

In addition to streamlining the production, 3D-printing also facilitates the exchange between research institutions. Developing and producing humanoid platforms for research requires technical and financial resources. The costs are multiplied with every new robot built as most components utilise customised and proprietary technology. Using standard, off-the-shelf, commercially available components with open-sourced designs alleviates these issues in both development and maintenance costs [91]. Obtaining a HUBO [15] requires hundreds of thousands of dollars, while the bill of materials for a similarly-sized open-source NimbRo-OP2(X) [39, 40•, 92] sums up to less than $10 \%$ of that price. The base capabilities of open platforms are naturally less than those of established products, but they offer a level of customisation that can be taken advantage of. As researchers can reuse already developed solutions, they are not faced with the problem of 'reinventing the wheel'. Resources can then be spent on perfecting certain parts of a proven design instead of focusing on overcoming the initial hurdle of building a complete robot. By involving 3D-printing in the process, the production and maintenance of robots no longer requires a separate machine shop for part production. A single $3 \mathrm{D}$ printer can not only reduce the production time but can also completely alleviate the dependence from a part manufacturer. Broken parts could be swiftly improved, tested, and put on common servers to share with other users, which would lead to an accelerated development of humanoid robotics as a whole.

\section{Conclusions}

Humanoid hardware design requires extensive knowledge and experience in mechatronics to produce a capable platform. We have presented a glimpse at state-of-the-art technologies that contribute to humanoid robotics today as well as novelties that will shape its future. The classical approach of highly rigid robots is gradually shifting towards compliant and dynamic ones for safer interaction with 


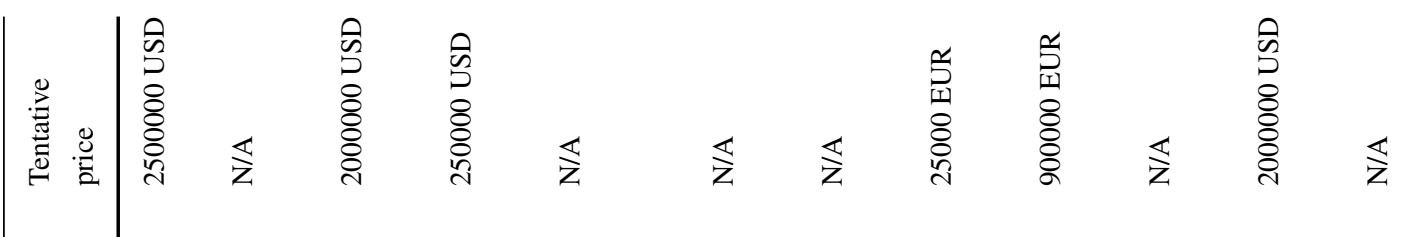

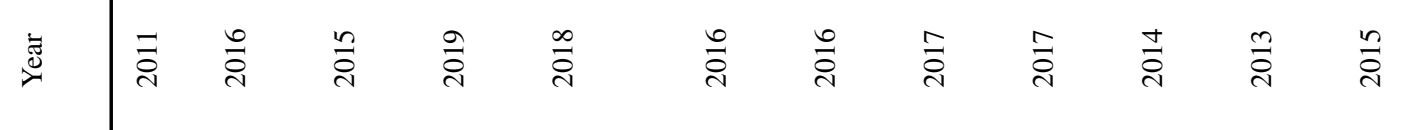

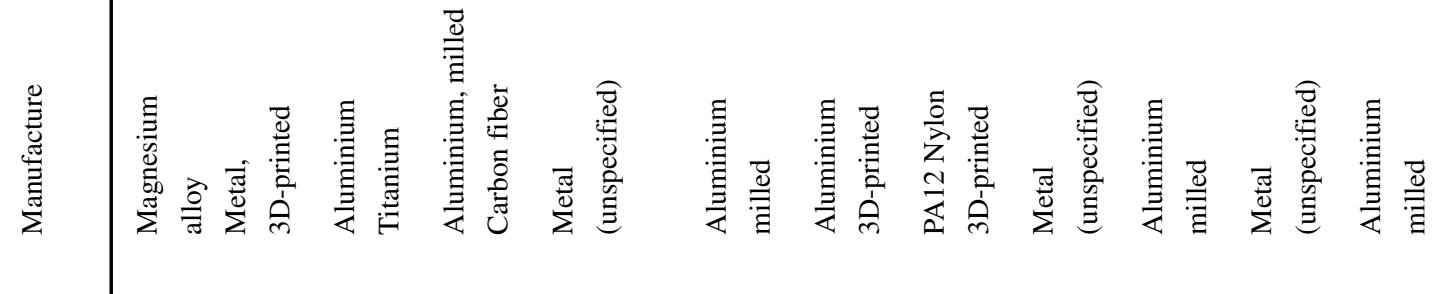

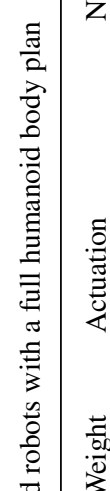

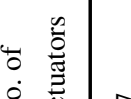

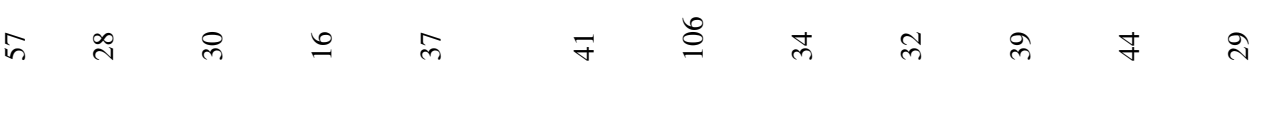

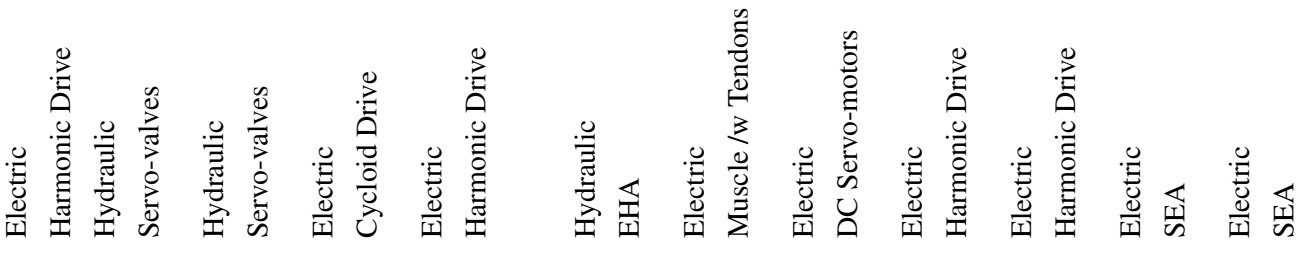

$$
\begin{aligned}
& \text { अ } \\
& \text { 嘉 }
\end{aligned}
$$

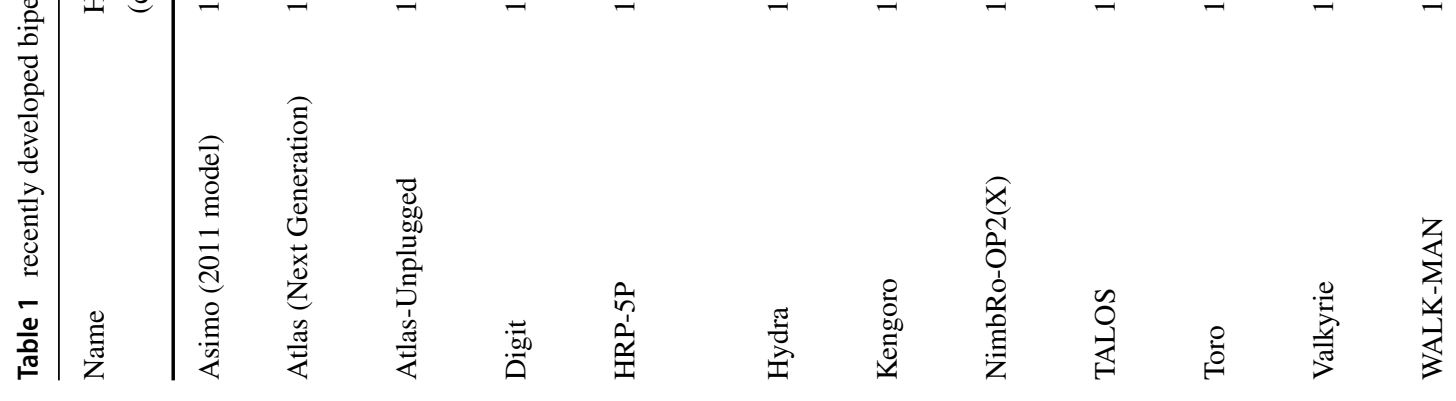


humans and more efficient locomotion. The discontinuation of ASIMO and the soaring popularity of ATLAS can be seen as a manifestation of this. Boston Dynamics are setting new, higher-than-ever standards for humanoid robotics, showing what can be achieved by breaking the typical design pattern. With easier access to rapid prototyping technologies, more full-scale humanoid robots are being built now than ever before. It will be exciting to see how all the technological advancements are utilised in future humanoid robots, and to what real tasks they can be applied to.

Funding Open Access funding enabled and organized by Projekt DEAL.

\section{Declarations}

Human and Animal Rights and Informed Consent This article does not contain any studies with human or animal subjects performed by any of the authors.

Conflict of Interests The authors declare no competing interests.

Open Access This article is licensed under a Creative Commons Attribution 4.0 International License, which permits use, sharing, adaptation, distribution and reproduction in any medium or format, as long as you give appropriate credit to the original author(s) and the source, provide a link to the Creative Commons licence, and indicate if changes were made. The images or other third party material in this article are included in the article's Creative Commons licence, unless indicated otherwise in a credit line to the material. If material is not included in the article's Creative Commons licence and your intended use is not permitted by statutory regulation or exceeds the permitted use, you will need to obtain permission directly from the copyright holder. To view a copy of this licence, visit http://creativecommons. org/licenses/by/4.0/.

\section{References}

Papers of particular interest, published recently, have been highlighted as:

- Of importance

$\bullet$ Of major importance

1. Behnke S. Humanoid robots-from fiction to reality? KI. 2008;22(4):5-9.

2. Froese R. Cube law, condition factor and weight-length relationships: history, meta-analysis and recommendations. J Appl Ichthyol. 2006;22(4):241-253.

3. Usherwood J, Gladman N. Why are the fastest runners of intermediate size? Contrasting scaling of mechanical demands and muscle supply of work and power. Biol Lett. 2020;16(10):20200579.

4. Shin H, Ishikawa T, Kamioka T, Hosoda K, Yoshiike T. Mechanistic properties of five-bar parallel mechanism for leg structure based on spring loaded inverted pendulum. In: 2019 IEEE-RAS 19th International conference on humanoid robots (Humanoids). IEEE; 2019. p. 320-327.

5. Playter R, Raibert MH. Control of a biped somersault in 3D. In: Proceedings of the IEEE/RSJ international conference on intelligent robots and systems. IEEE.; 1992. p. 582-589.
6. Hubicki C, Grimes J, Jones M, Renjewski D, Spröwitz A, Abate A, et al. Atrias: design and validation of a tetherfree 3d-capable spring-mass bipedal robot. Int J Robot Res. 2016;35(12):1497-1521.

7. Abate AM. Mechanical design for robot locomotion. 2018.

8. Shigemi S, Goswami A, Vadakkepat P. ASIMO and humanoid robot research at Honda. Humanoid Robotics: A Reference. p. 55-90. 2018.

9. Yokoi K, Kanehiro F, Kaneko K, Kajita S, Fujiwara K, Hirukawa H. Experimental study of humanoid robot HRP-1S. Int J Robot Res. 2004.

10. Hirukawa H, Kanehiro F, Kaneko K, Kajita S, Fujiwara K, Kawai Y, et al. Humanoid robotics platforms developed in HRP. Rob Auton Syst. 2004.

11. Kaneko K, Harada K, Kanehiro F, Miyamori G, Akachi K. Humanoid robot HRP-3. In: IEEE/RSJ International conference on intelligent robots and systems (IROS); 2008.

12. Kaneko K, Kanehiro F, Morisawa M, Akachi K, Miyamori G, Hayashi A, et al. Humanoid robot HRP-4-humanoid robotics platform with lightweight and slim body. In: IEEE/RSJ International Conference on Intelligent Robots and Systems (IROS). 2011.

13. Kaneko K, Kanehiro F, Morisawa M, Miura K, Nakaoka S, Kajita S. Cybernetic Human HRP-4C. In: Proceedings of 9th IEEE-RAS Int. Conf. on Humanoid Robotics (Humanoids), 2009; p. 7-14.

14. Kaneko K, Kaminaga H, Sakaguchi T, Kajita S, Morisawa M, Kumagai I, et al. Humanoid robot HRP-5P: An electrically actuated humanoid robot with high-power and wide-range joints. IEEE Robot Autom Lett. 2019;4(2):14311438.

15. Park IW, Kim JY, Lee J, Oh JH. Mechanical design of the humanoid robot platform, HUBO. Adv Robot. 2007;21(11):1305-1322.

16. Robotics P. Reem-c. Data de consulta. 18. 2015.

17. Stasse O, Flayols T, Budhiraja R, Giraud-Esclasse K, Carpentier J, Mirabel J. TALOS: A new humanoid research platform targeted for industrial applications. In: 2017 IEEERAS 17th International conference on humanoid robotics (Humanoids). IEEE; 2017. p. 689-695.

18. Tolani D, Goswami A, Badler NI. Real-time inverse kinematics techniques for anthropomorphic limbs. Graph Models. 2000;62(5):353-388.

19. Hirose M, Ogawa K. Honda humanoid robots development. Philos Trans A Math Phys Eng Sci. 2007;365(1850):11-19.

20. Ceccarelli M, Russo M, Morales-Cruz C. Parallel architectures for humanoid robots. Robotics. 2020;9(4):75.

21. Tazaki Y. Parallel link-based light-weight leg design for bipedal robots. In: 2019 IEEE-RAS 19th International conference on humanoid robots (Humanoids). IEEE; 2019. p. 565-571.

22. Lee B, Knabe C, Orekhov V, Hong D. Design of a humanlike range of motion hip joint for humanoid robots. In: ASME 2014 International design engineering technical conferences and computers and information in engineering conference. American society of mechanical engineers digital collection; 2014.

23. Mghames S, Catalano MG, Bicchi A, Grioli G. A spherical active joint for humanoids and humans. IEEE Robot Autom Lett. 2019;4(2):838-845.

24. Lahr D, Orekhov V, Lee B, Hong D. Early developments of a parallelly actuated humanoid, SAFFiR. In: ASME 2013 International design engineering technical conferences and computers and information in engineering conference. American society of mechanical engineers digital collection; 2013. 
25. Kakiuchi Y, Kamon M, Shimomura N, Yukizaki S, Takasugi $\mathrm{N}$, Nozawa $\mathrm{S}$, et al. Development of life-sized humanoid robot platform with robustness for falling down, long time working and error occurrence. In: 2017 IEEE/RSJ International conference on intelligent robots and systems (IROS). IEEE; 2017. p. 689-696.

26. Negrello F, Garabini M, Catalano MG, Kryczka P, Choi W, Caldwell DG, et al. Walk-man humanoid lower body design optimization for enhanced physical performance. 2016. In: IEEE International conference on robotics and automation (ICRA). IEEE; 2016. p. 1817-1824.

27. Kamioka T, Kaneko H, Kuroda M, Tanaka C, Shirokura $\mathrm{S}$, Takeda $\mathrm{M}$, et al. Push recovery strategy of dynamic gait transition between walking, running and hopping. Int J Human Robot. 2019;16(03):1940001.

28. Lohmeier S, Buschmann T, Schwienbacher M, Ulbrich H, Pfeiffer F. Leg design for a humanoid walking robot. In: 2006 6th IEEE-RAS International conference on humanoid robots. IEEE; 2006. p. 536-541.

29. Tomishiro K, Sato R, Harada Y, Ming A, Meng F, Liu H, et al. Design of robot leg with variable reduction ratio crossed four-bar linkage mechanism. In: 2019 IEEE/RSJ International conference on intelligent robots and systems (IROS). IEEE; 2019. p. 4333-4338.

30. Honda Motor Co Ltd. Legged mobile robot; Patent JP 201394943 A. 2013.

31. Englsberger J, Werner A, Ott C, Henze B, Roa MA, Garofalo G, et al. Overview of the torque-controlled humanoid robot TORO. In: 2014 IEEE-RAS International conference on humanoid robots. IEEE; 2014. p. 916-923.

32. Zhou C, Tsagarakis N. On the comprehensive kinematics analysis of a humanoid parallel ankle mechanism. J Mech Robot. 2018;10(5).

33. Hancock PJ. Adaptive torque control of a Novel 3D-Printed Humanoid Leg. Virginia Tech. 2020.

34. Otani T, Iizuka A, Takamoto D, Motohashi H, Kishi $\mathrm{T}$, Kryczka P, et al. New shank mechanism for humanoid robot mimicking human-like walking in horizontal and frontal plane. In: 2013 IEEE international conference on robotics and automation. IEEE; 2013. p. 667-672.

35. Gim KG, Kim J, Yamane K. Design of a serial-parallel hybrid leg for a humanoid robot. In: 2018 IEEE International conference on robotics and automation (ICRA). IEEE; 2018. p. 6076-6081.

36. Gim KG, Kim J, Yamane K. Design and fabrication of a bipedal robot using serial-parallel hybrid leg mechanism. In: 2018 IEEE/RSJ International conference on intelligent robots and systems (IROS). IEEE; 2018. p. 5095-5100.

37. Robotis Ltd. ROBOTIS OP3; 2021. Available from: https:// emanual.robotis.com/docs/en/platform/op3/introduction/.

38. Kajita S, Kanehiro F, Kaneko K, Yokoi K, Hirukawa H. The 3D linear inverted pendulum mode: a simple modeling for a biped walking pattern generation. In: Proceedings 2001 IEEE/RSJ International conference on intelligent robots and systems. Expanding the societal role of robotics in the the next millennium (Cat. No. 01CH37180). IEEE; 2001. p. 239-246.

39. Ficht G, Allgeuer P, Farazi H, Behnke S. NimbRo-OP2: Grown-up 3D printed open humanoid platform for research. In: 2017 IEEE-RAS 17th International conference on humanoid robotics (Humanoids). IEEE; 2017. p. 669-675.

40. Ficht G, Farazi H, Brandenburger A, Rodriguez D, Pavlichenko D, Allgeuer P, et al. NimbRo-OP2X: Adultsized open-source 3D printed humanoid robot. In: 2018 IEEE-RAS 18th International conference on humanoid robots (Humanoids). IEEE; 2018. p. 1-9. This article provides an overview of a large bipedal humanoid robot, with a completely 3D-printed structure and off-the-shelf components, which greatly reduces the acquisition cost.

41. Saab W, Rone WS, Ben-Tzvi P. Robotic modular leg: design, analysis, and experimentation. J Mech Robot. 2017;9(2).

42. Han J, Hong D. Development of a full-sized bipedal humanoid robot utilizing spring assisted parallel four-bar linkages with synchronized actuation. In: International design engineering technical conferences and computers and information in engineering conference; 2011. p. 799-806.

43. Sharbafi MA, Rode C, Kurowski S, Scholz D, Möckel $\mathrm{R}$, Radkhah $\mathrm{K}$, et al. A new biarticular actuator design facilitates control of leg function in BioBiped3. Bioinspir Biomim. 2016;11(4):046003.

44. Roozing W, Ren Z, Tsagarakis NG. An efficient leg with series-parallel and biarticular compliant actuation: design optimization, modeling, and control of the eLeg. Int J Robot Res. 2019:0278364919893762.

45. Schumacher C, Sharbafi M, Seyfarth A, Rode C. Biarticular muscles in light of template models, experiments and robotics: a review. J Roy Soc Interface. 2020;17(163): 20180413. This study provides a comprehensive overview on how biarticulation affects bipedal locomotion based on human data, segmented template models and selected robots.

46. Schütz S, Nejadfard A, Mianowski K, Vonwirth P, Berns K. CARL-A compliant robotic leg featuring mono-and biarticular actuation. In: 2017 IEEE-RAS 17th International conference on humanoid robotics (Humanoids). IEEE; 2017. p. 289-296.

47. Ananthanarayanan A, Azadi M, Kim S. Towards a bioinspired leg design for high-speed running. Bioinspir Biomim. 2012;7(4):046005.

48. Feldmann S, Bruckmann T, Schramm D. Simulation of a cable-driven actuation concept for a humanoid robot prototype. In: 2014 IEEE/ASME 10th International conference on mechatronic and embedded systems and applications (MESA). IEEE; 2014. p. 1-6.

49. Nakanishi Y, Asano Y, Kozuki T, Mizoguchi H, Motegi Y, Osada M, et al. Design concept of detail musculoskeletal humanoid Kenshiro-toward a real human body musculoskeletal simulator. In: 2012 12th IEEE-RAS International conference on humanoid robots (Humanoids 2012). IEEE; 2012. p. 1-6.

50. Asano Y, Kozuki T, Ookubo S, Kawamura M, Nakashima S, Katayama T, et al. Human mimetic musculoskeletal humanoid Kengoro toward real world physically interactive actions. In: 2016 IEEE-RAS 16th International conference on humanoid robots (Humanoids). IEEE; 2016. p. 876-883.

51. Radford NA, Strawser P, Hambuchen K, Mehling JS, Verdeyen WK, Donnan AS, et al. Valkyrie: Nasa's first bipedal humanoid robot. J Field Robot. 2015;32(3):397-419.

52. Tsagarakis NG, Caldwell DG, Negrello F, Choi W, Baccelliere L, Loc VG, et al. Walk-man: a high-performance humanoid platform for realistic environments. J Field Robot. 2017;34(7):1225-1259.

53. Negrello F, Garabini M, Catalano MG, Malzahn J, Caldwell DG, Bicchi A, et al. A modular compliant actuator for emerging high performance and fall-resilient humanoids. In: 2015 IEEE-RAS 15th International conference on humanoid robots (Humanoids). IEEE; 2015. p. 414-420.

54. Martins LT, Tatsch C, Maciel EH, Henriques RVB, Gerndt R, da Guerra RS. Polyurethane-based modular series elastic upgrade to a robotics actuator. In: Robot soccer world cup; 2015. p. 347-355.

55. Katz BG. A low cost modular actuator for dynamic robots. Massachusetts Institute of Technology. 2018. This thesis 
provides a design template for compact and powerful QDD actuators.

56. Katz B, Di Carlo J, Kim S. Mini cheetah: a platform for pushing the limits of dynamic quadruped control. In: 2019 International conference on robotics and automation (ICRA). IEEE; 2019. p. 6295-6301.

57. Ramos J, Katz B, Chuah MYM, Kim S. Facilitating modelbased control through software-hardware co-design. 2018. In: IEEE international conference on robotics and automation (ICRA). IEEE; 2018. p. 566-572.

58. Kato I, Ohteru S, Kobayashi H, Shirai K, Uchiyama A. Information-power machine with senses and limbs. In: On theory and practice of robots and manipulators. Springer; 1974. p. 11-24.

59. Boston Dynamics. Available from: https://www. bostondynamics.com/; 2021.

60. Nelson G, Saunders A, Neville N, Swilling B, Bondaryk J, Billings D, et al. Petman: A humanoid robot for testing chemical protective clothing. J Robot Soc Jpn. 2012;30(4):372-377.

61. Nelson G, Saunders A, Playter R. The petman and atlas robots at boston dynamics. Humanoid Robotics: a Reference. p. 169-186. 2019.

62. Boston Dynamics. Available from: https://www.youtube.com/ watch?v=rVlhMGQgDkY. 2016.

63. Boston Dynamics. More Parkour Atlas. 2019. Available from: https://www.youtube.com/watch?v=_sBBaNYex3E.

64. Boston Dynamics. Do You Love Me?. 2020. Available from: https://www.youtube.com/watch? $=$ =fn3KWM1kuAw.

65. Johnson M, Shrewsbury B, Bertrand S, Calvert D, Wu T, Duran D, et al. Team IHMC's lessons learned from the DARPA Robotics Challenge: Finding data in the rubble. J Field Robot. 2017;34(2):241-261.

66. Kaminaga H, Ko T, Masumura R, Komagata M, Sato $\mathrm{S}$, Yorita S, et al. Mechanism and control of whole-body electro-hydrostatic actuator driven humanoid robot hydra. In: International symposium on experimental robotics. Springer; 2016. p. 656-665.

67. Hyon SH, Suewaka D, Torii Y, Oku N. Design and experimental evaluation of a fast torque-controlled hydraulic humanoid robot. IEEE/ASME Trans Mechatron. 2016;22(2):623-634.

68. Kaminaga H, Otsuki S, Nakamura Y. Development of highpower and backdrivable linear electro-hydrostatic actuator. In: 2014 IEEE-RAS International conference on humanoid robots. IEEE; 2014. p. 973-978.

69. Li X, Zhou H, Zhang S, Feng H, Fu Y. WLR-II a hose-less hydraulic wheel-legged robot. In: 2019 IEEE/RSJ International conference on intelligent robots and systems (IROS). IEEE; 2019. p. 4339-4346.

70. Kim U, Lee DH, Kim YB, Seok DY, Choi HR. A novel sixaxis force/torque sensor for robotic applications. IEEE/ASME Trans Mechatron. 2016;22(3):1381-1391.

71. Noh Y, Bimbo J, Sareh S, Wurdemann H, Fraś J, Chathuranga DS, et al. Multi-axis force/torque sensor based on simply-supported beam and optoelectronics. Sensors. 2016;16(11):1936.

72. Atkeson CG, Babu B, Banerjee N, Berenson D, Bove C, Cui $\mathrm{X}$, et al. What happened at the darpa robotics challenge, and why. submitted to the DRC Finals Special Issue of the Journal of Field Robotics. 2016.

73. Zou L, Ge C, Wang ZJ, Cretu E, Li X. Novel tactile sensor technology and smart tactile sensing systems: A review. Sensors. 2017;17(11):2653.

74. Mittendorfer P, Cheng G. Humanoid multimodal tactilesensing modules. IEEE Trans Robot. 2011;27(3):401-410.

75. Mittendorfer P, Cheng G. Integrating discrete force cells into multi-modal artificial skin. In: 2012 12th IEEE-RAS
International conference on humanoid robots (Humanoids 2012). IEEE; 2012. p. 847-852.

76. Mittendorfer P, Yoshida E, Cheng G. Realizing whole-body tactile interactions with a self-organizing, multi-modal artificial skin on a humanoid robot. Adv Robot. 2015;29(1):51-67.

77. Kaboli M, Feng D, Cheng G. Active tactile transfer learning for object discrimination in an unstructured environment using multimodal robotic skin. Int $\mathrm{J}$ Hum Robot. 2018;15(01):1850001.

78. Leboutet Q, Dean-Leon E, Bergner F, Cheng G. Tactile-based whole-body compliance with force propagation for mobile manipulators. IEEE Trans Robot. 2019;35(2):330-342.

79. Kobayashi T, Dean-Leon E, Guadarrama-Olvera JR, Bergner F, Cheng G. Multi-contacts force-reactive walking control during physical human-humanoid interaction. In: 2019 IEEERAS 19th international conference on humanoid robots (Humanoids). IEEE; 2019. p. 33-39.

80. Rogelio Guadarrama Olvera J, Leon ED, Bergner F, Cheng G. Plantar tactile feedback for biped balance and locomotion on unknown terrain. Int J Hum Robot. 2020;17(01):1950036. This study shows the effect of extra modalities obtained with robot skin in balanced walking on obstacles, in comparison to typically used force-torque sensors.

81. Parmiggiani A, Maggiali M, Natale L, Nori F, Schmitz A, Tsagarakis $\mathrm{N}$, et al. The design of the iCub humanoid robot. Int J Hum Robot. 2012;9(04):1250027.

82. Metta G, Natale L, Nori F, Sandini G, Vernon D, Fadiga L, et al. The iCub humanoid robot: an open-systems platform for research in cognitive development. Neural Netw. 2010;23(89):1125-1134.

83. Lohmeier S, Buschmann $\mathrm{T}$, Ulbrich $\mathrm{H}$. Humanoid robot LOLA. In: 2009 IEEE International conference on robotics and automation. IEEE; 2009. p. 775-780.

84. Jones R, Haufe P, Sells E, Iravani P, Olliver V, Palmer $\mathrm{C}$, et al. RepRap-the replicating rapid prototyper. Robotica. 2011;29(1):177-191.

85. Lapeyre M, Rouanet P, Grizou J, Nguyen S, Depraetre F, Le Falher A, et al. Poppy project: open-source fabrication of $3 \mathrm{D}$ printed humanoid robot for science, education and art. Digit Intell. 2014;2014:6.

86. Allgeuer P, Farazi H, Schreiber M, Behnke S. Child-sized 3D printed igus humanoid open platform. In: 2015 IEEERAS 15th International conference on humanoid robots (Humanoids). IEEE; 2015. p. 33-40.

87. Wu L, Larkin M, Potnuru A, Tadesse Y. HBS-1: a modular child-size 3D printed humanoid. Robotics. 2016;5(1): 1 .

88. Junk S, Klerch B, Hochberg U. Additive manufacturing of a topology optimized lightweight part of a humanoid robot. In: Sim-AM 2019: II International conference on simulation for additive manufacturing. CIMNE; 2019. p. 142-153.

89. Klemm V, Morra A, Salzmann C, Tschopp F, Bodie K, Gulich L, et al. Ascento: a two-wheeled jumping robot. In: 2019 International conference on robotics and automation (ICRA). IEEE; 2019. p. 7515-7521.

90. Buchanan C, Gardner L. Metal 3D-printing in construction: a review of methods, research, applications, opportunities and challenges. Eng Struct. 2019;180:332-348.

91. Allgeuer P, Farazi H, Ficht G, Schreiber M, Behnke S. The igus humanoid open platform. KI-Kü,nstliche Intelligenz. 2016;30(3):315-319.

92. Ficht G, Farazi H, Rodriguez D, Pavlichenko D, Allgeuer P, Brandenburger A, et al. Nimbro-OP2X: affordable adult-sized 3d-printed open-source humanoid robot for research. Int J Hum Robot. 2020;17(05):2050021.

Publisher's Note Springer Nature remains neutral with regard to jurisdictional claims in published maps and institutional affiliations. 